\title{
Colon cancers carrying BRAF V600E and $\beta$-catenin T41A activating mutations are resistant to numerous common anticancer drugs
}

\author{
GUANGZHONG XU ${ }^{1,2}, \mathrm{KAI} \mathrm{LI}^{1}$, NENGWEI ZHANG ${ }^{1}$, BIN ZHU $^{1}$, GUOSHENG FENG ${ }^{2}$ and QING FAN ${ }^{1}$ \\ ${ }^{1}$ Department of General Surgery, Beijing Shijitan Hospital, Capital Medical University, Beijing 100038; \\ ${ }^{2}$ Department of General Surgery, Beijing Chao-Yang Hospital, Capital Medical University, Beijing 100020, P.R. China
}

Received January 19, 2017; Accepted October 26, 2017

DOI: $10.3892 / \mathrm{ol} .2018 .7856$

\begin{abstract}
Colorectal cancer is a common malignancy with a high prevalence and associated mortality rate. However, the preclinical tools currently used for drug development are insufficient. The aim of the present study was to establish and characterize a specific patient-derived colon cancer xenograft (PDCCX) mouse model for drug testing. Primary colon tumors were obtained from 10 patients by surgical resection, and tumor tissues were subsequently grafted into nude mice followed by consecutive passages. Primary tumors and xenograft tumors were collected and processed for DNA sequencing, histological evaluation and immunohistochemical staining. The responses of fifth-generation PDCCX mice to 5-fluorouracil, oxaliplatin, and cetuximab were assessed. Two PDCCX cell lines were successfully established. The histology and protein expression levels of SMAD family member 3, epidermal growth factor receptor, c-MET, caudal type homeobox 2 , E-cadherin and $\beta$-catenin in the xenograft tumors were consistently maintained from the primary cancer tissues. BRAF V600E and $\beta$-catenin T41A double mutations were identified in one cell line, and were associated with a lack of response to 5-fluorouracil, oxaliplatin and cetuximab treatment. This PDCCX cell line may provide a reliable tool for preclinical evaluation of the efficacy of novel therapies that may target the BRAF V600E and $\beta$-catenin mutations.
\end{abstract}

Correspondence to: Dr Nengwei Zhang, Department of General Surgery, Beijing Shijitan Hospital, Capital Medical University, 10 Tieyi Road, Beijing 100038, P.R. China

E-mail: zhangnw1@sohu.com

Dr Guosheng Feng, Department of General Surgery, Beijing Chao-Yang Hospital, Capital Medical University, 8 Gongren Tiyuchangnan Road, Beijing 100020, P.R. China

E-mail: guosheng_feng@sina.com

Key words: patient-derived cancer xenograft, colon cancer, BRAF $\mathrm{V} 600 \mathrm{E}$ mutation, $\beta$-catenin activating mutation, drug resistance

\section{Introduction}

Colorectal cancer (CRC) is a common malignancy with a high prevalence and associated mortality rate (1). The etiology and pathogenetic changes of CRC are complex and heterogeneous. The risk factors for $\mathrm{CRC}$ include a diet rich in unsaturated fats, consumption of red meat, excessive total energy intake, excessive alcohol consumption, and inherited and somatic mutations $(2,3)$. Significant progress has been made in identifying genetic changes associated with the pathogenesis of CRC $(3,4)$. Although adenomatous polyposis coli (APC) is the most frequently mutated gene in CRC, mutations in P53, KRAS, NRAS, phosphatidylinositol-4,5-bisphosphate 3 -kinase catalytic subunit $\alpha$, F-box and WD repeat domain-containing protein 7, SMAD2, SMAD 4, transcription factor 7-like 2 (TCF7L2) and $\beta$-catenin (CTNNB1) are also frequently observed in this cancer type $(3,4)$. Additionally, BRAF mutations are present in $5-8 \%$ of CRC cases, with a single missense V600E mutation accounting for $80 \%$ of these (5). BRAF mutations are often associated with aggressiveness, poor differentiation and resistance to therapy in CRC (4). CRC patients with BRAF V600E mutations exhibit a poor prognosis and a poor response to panitumumab and cetuximab, monoclonal antibodies targeting the epidermal growth factor receptor (EGFR/ERBB1) (6).

The Wnt/ $\beta$-catenin pathway serves an important role in $\mathrm{CRC}$, and numerous pathway components, including APC, axin, TCF7L2 and $\beta$-catenin, are mutated in CRC $(3,7)$. Additionally, the activated $\mathrm{Wnt} / \beta$-catenin pathway interacts with a number of other signaling pathways and regulators in modulating oncogenic processes (8-12), which further complicates the regulation and functions of the $\mathrm{Wnt} / \beta$-catenin pathway in cancer development.

Until now, human cancer cell lines and cancer cell xenograft mouse models have been irreplaceable tools used in preclinical cancer drug development. However, it has been demonstrated that cancer cell lines often lose the biological properties of the original cancer, including heterogeneity, genetic characteristics, migratory and metastatic abilities, the maintenance of a stem cell population, and dependency on embryonic signaling pathways (13-17). These deficiencies cause significant setbacks in cancer therapeutic development 
and result in financial and human losses. The emergence of patient-derived cancer xenograft (PDX) models may provide a reliable alternative to cancer cell xenografts for the development of cancer drugs (18-20). However, there have been no previous reports on the establishment of a CRC PDX model; thus, the present study aimed to establish and characterize a specific CRC PDX mouse model for drug testing.

\section{Materials and methods}

Patients and tissues. The study protocol was approved by the Institutional Review Board of Capital Medical University (Beijing, China). Written informed consent was obtained from each participant. Colon cancer tissues were obtained from 10 patients ( 7 male, 3 female; age range, 38-72 years; average age, 57.3 years) who had undergone surgical resection at Beijing Shijitan Hospital or Beijing Chao-Yang Hospital (Beijing, China) between February 2015 and June 2015 (Table I). Each primary cancer tissue was divided into three parts: One part for in vivo grafting; one part for processing into formalin-fixed, paraffin-embedded (FFPE) tissue blocks; and one part for genomic DNA extraction.

Establishment of patient-derived colon cancer xenograft (PDCCX) model. Surgically removed colon cancer tissues (F0) were immediately placed into $4^{\circ} \mathrm{C}$ Hank's balanced salt solution (Thermo Fisher Scientific, Inc., Waltham, MA, USA) with $100 \mathrm{U} / \mathrm{ml}$ penicillin and $100 \mu \mathrm{g} / \mathrm{ml}$ streptomycin and transported to the animal facility within $2 \mathrm{~h}$. Necrotic tissue and blood were removed from the cancer tissues prior to the tissue being cut into 1 - to $2-\mathrm{mm}$ pieces and subcutaneously implanted into the right hind flanks of 5 immune-compromised nude mice (6 weeks old; male to female, 1:1; $20 \pm 2$ g; Changzhou Cavens Laboratory Animal Co., Jiangsu, China) per patient tumor tissue. Mice were maintained in a germ-free facility at $22-25^{\circ} \mathrm{C}, 55 \%$ humidity, $12 \mathrm{~h}$ light/dark cycle and free access to food and water. The mice were routinely monitored for discomfort, distress or pain. Once the xenografted tumors reached $\sim 500 \mathrm{~mm}^{3}$, the tumor-bearing mice were sacrificed and the tumors were resected and separated into three parts as for the primary tumors. Following 5 generations (F5) of consecutive xenografts, a PDCCX model was considered to have been established. The protocols for all animal experiments were reviewed and approved by The Committee for Laboratory Animal Care and Usage of Capital Medical University.

Immunohistochemistry (IHC). IHC was used to evaluate six biomarkers at the protein level. Tumor tissues were fixed in 10\% neutral-buffered formalin (Wuxi Zhanwan Chemicals, Yixing, China; http://www.yxzw.com) at room temperature for 1 week prior to sectioning. The $4-\mu \mathrm{m}$-thick FFPE sections were deparaffinized in xylene twice ( 5 min each) and rehydrated in a descending ethanol series. Antigen retrieval was performed in a pressure cooker using sodium citrate buffer (10 mM sodium citrate, $0.05 \%$ Tween $20, \mathrm{pH} 6.0$ ). Endogenous peroxidase activity was blocked by incubation with $3 \% \mathrm{H}_{2} \mathrm{O}_{2}$ in PBS at room temperature for 5-10 $\mathrm{min}$. The sections were incubated with normal goat serum (Jackson ImmunoResearch Laboratories, Inc., West Grove, PA) for $30 \mathrm{~min}$ at room temperature, followed by incubation with primary antibodies against
Table I. Characteristics of primary colon cancers.

\begin{tabular}{ll}
\hline Variable & No. of cases \\
\hline Dukes staging & 1 \\
A & 3 \\
B & 6 \\
C & \\
Differentiation level & 2 \\
Poor & 5 \\
Medium & 3 \\
High & \\
Tumor type & 5 \\
Ulcerated & 3 \\
Infiltrative & 2 \\
Elevated & \\
Associated pathological symptoms & 3 \\
Intestinal Metaplasia & 4 \\
Mucosal atrophy & 3 \\
Neither & \\
\hline
\end{tabular}

$\beta$-catenin (1:400; cat. no. 9562), ERBB1 (1:50; cat. no. 4267), c-MET (1:250; cat. no. 8198), caudal type homeobox 2 (CDX2; 1:1,000; cat. no. 12306), E-cadherin (1:100; cat. no. 14472) (all from Cell Signaling Technology, Inc., Danvers, MA, USA) and SMAD3 (1:100; cat. no. ab40854; Abcam, Cambridge, UK) overnight at $4^{\circ} \mathrm{C}$. Following two rinses (5 min each) in PBS, the sections were incubated with a biotin-conjugated goat anti-rabbit immunoglobulin (Ig)G (1:200; cat. no. BA1003; Boster Biological Technology, Pleasanton, CA, USA) following all primary antibody incubations except those of E-cadherin, for which a goat anti-mouse IgG secondary antibody was used (1:200; cat. no. BA1001; Boster Biological Technology) at room temperature for $20 \mathrm{~min}$ and rinsed twice (5 min each) in PBS. Sections were then incubated with streptavidin-biotin complex reagent (horseradish peroxidase-conjugated anti-Human IgG SABC kit; cat. no. SA1024; Boster Biological Technology) at $37^{\circ} \mathrm{C}$ for $20 \mathrm{~min}$, rinsed four times for $5 \mathrm{~min}$ each, and developed with a Pierce DAB kit (Thermo Fisher Scientific, Inc.). The sections were counterstained with hematoxylin, dehydrated in an ethanol gradient, cleared in xylene and mounted. The slides were observed and imaged (x100, magnification) using an Olympus IX71 fluorescence microscope (Olympus Corporation, Tokyo, Japan).

Mutation analyses. Genomic DNA was extracted from primary tumors (F0) and F5 xenograft tumors using a DNeasy Blood $\&$ Tissue kit (Qiagen China Co., Ltd., Shanghai, China). The genomic DNA was amplified and sequenced with the primers presented in Table II. Polymerase chain reaction (PCR) was performed using a Thermal Cycler (Thermo Fisher Scientific, Inc.) using Phusion High-Fidelity PCR Master Mix (New England BioLabs, Inc., Ipswich, MA, USA) with the following conditions: $95^{\circ} \mathrm{C}$ for $5 \mathrm{~min}$ followed by 30 cycles of $95^{\circ} \mathrm{C}$ for $30 \mathrm{sec}, 58^{\circ} \mathrm{C}$ for $30 \mathrm{sec}$, and $68^{\circ} \mathrm{C}$ for $30 \mathrm{sec}$. The PCR products were separated on agarose gels $(0.8 \%)$ and sequenced by Sangon Biotech Co., 


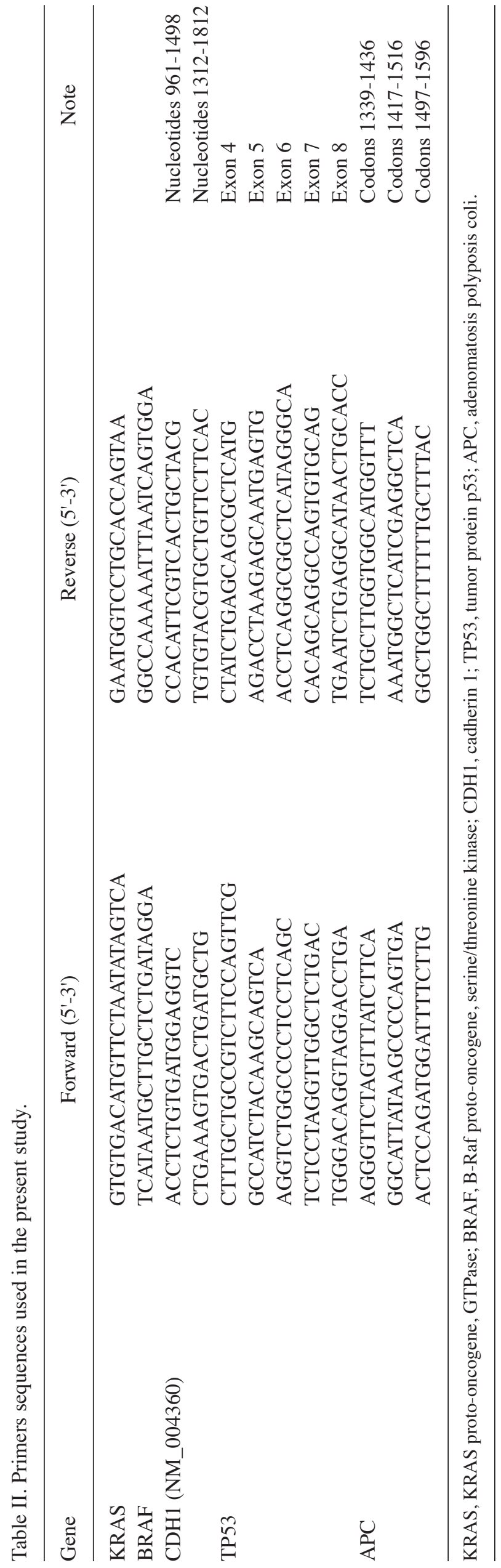

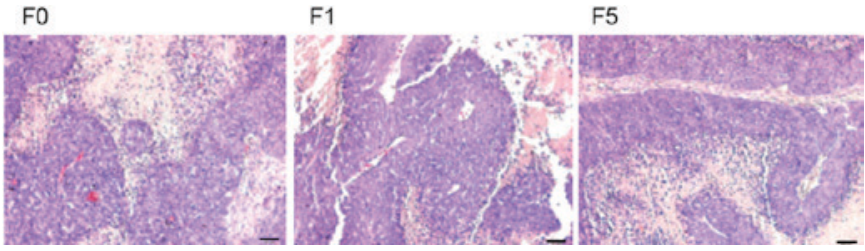

Figure 1. Patient-derived colon cancer xenograft mouse model maintained the histology of the primary cancer. Primary colon cancer (F0) and engrafted tumors (F1 and F5) exhibited the same histology following hematoxylin staining. Scale bar $=50 \mu \mathrm{m}$. F, generation.

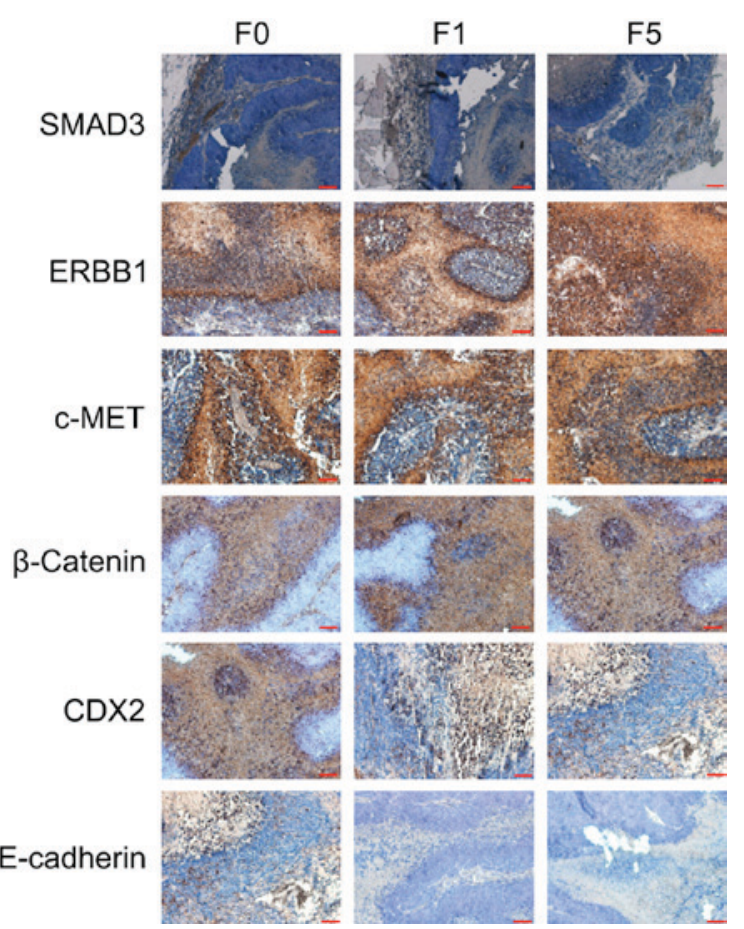

Figure 2. Patient-derived colon cancer xenograft tumors (F1 and F5) exhibited the same positivity and expression levels of protein biomarkers as the parental human cancer (F0). Immunohistochemical staining demonstrated common expression of SMAD3, ERBB1, c-MET, CDX2, E-cadherin and $\beta$-catenin between the primary cancer (F0) and the engrafted tumors (F1 and F5). Scale bar $=50 \mu \mathrm{m} . \mathrm{F}$, generation; ERBB1, epidermal growth factor receptor; $\mathrm{CDX} 2$, caudal type homeobox 2.

Ltd. (Shanghai, China). The mutations were analyzed and visualized using FinchTV (version 1.4.0; Geospiza; PerkinElmer, Waltham, MA, USA).

Drug sensitivity assessment. Once the F5 xenograft tumors reached a size of $\sim 150 \mathrm{~mm}^{3}$, tumor-bearing mice were intraperitoneally administered with saline, cetuximab $(1 \mathrm{mg} / \mathrm{kg}$ every 3 days, 5 times in total), 5 -fluorouracil (5-FU; $10 \mathrm{mg} / \mathrm{kg}$ for 5 days) or oxaliplatin ( $3 \mathrm{mg} / \mathrm{kg}$ for 5 days). The tumor sizes were measured every 5 days.

Statistical analysis. The data are expressed as the mean \pm standard error of the mean. Statistical analyses were performed using GraphPad Prism 6 (GraphPad Software, Inc., La Jolla, CA, USA). The differences among groups were analyzed using one-way analysis of variance followed by Tukey's post hoc test. $\mathrm{P}<0.05$ was considered to indicate a statistically significant difference. 
A

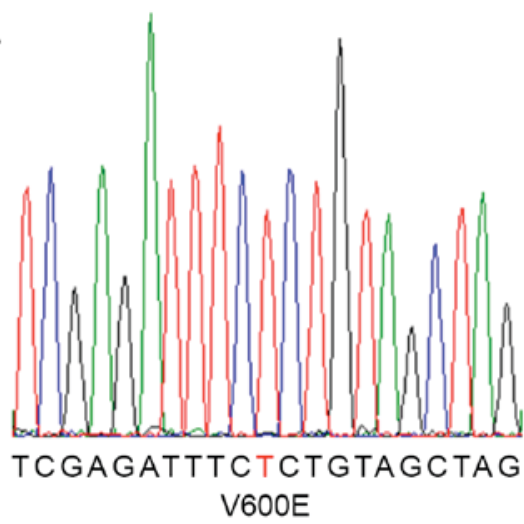

B

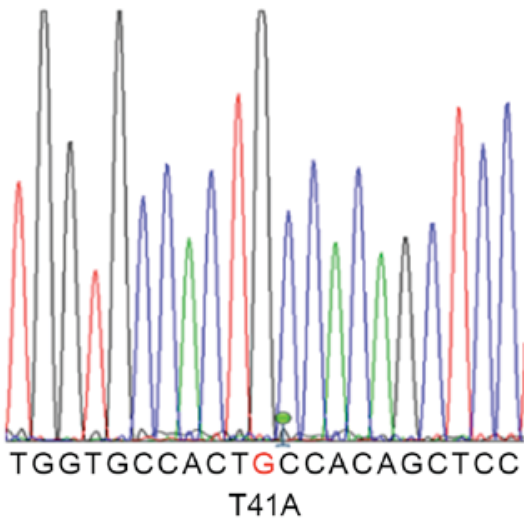

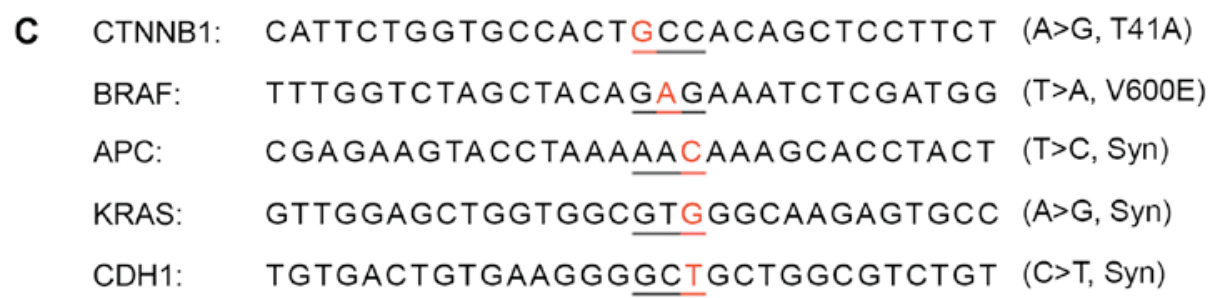

Figure 3. BRAF V600E and $\beta$-catenin T41A double mutations were identified in one PDCCX line. The point mutations (A) BRAF V600E and (B) $\beta$-catenin T41A were identified in the primary cancer and PDCCX tumors (F1 and F5). (C) A summary of mutations identified in this specific colon cancer and its derived PDCCX line. PDCCX, patient-derived colon cancer xenograft; F, generation; CTNNB1, $\beta$-catenin; APC, adenomatous polyposis coli; CDH1, cadherin 1; Syn, synonymous mutation.
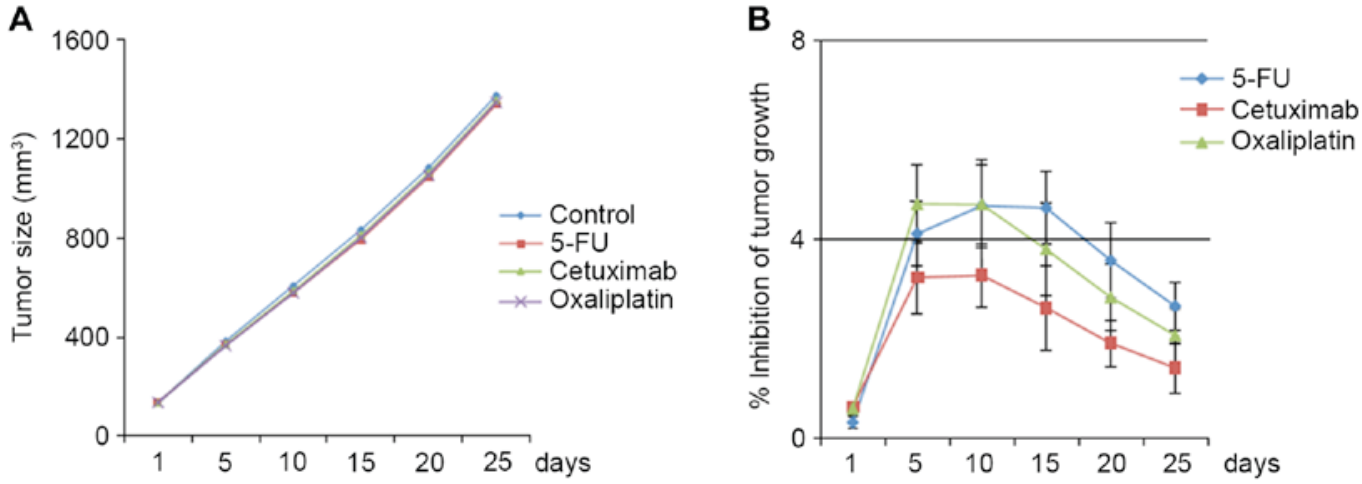

Figure 4. BRAF V600E- and CTNNB1 T41A-bearing colon cancer was resistant to 5-FU, oxaliplatin and cetuximab. (A) Growth of PDCCX tumors carrying BRAF V600E and CTNNB1 T41A mutations following the treatment of tumor-bearing mice ( $\mathrm{n}=5$ ) with saline, 5-FU, oxaliplatin or cetuximab. (B) Inhibition of tumor growth by 5 -FU, oxaliplatin and cetuximab, relative to that in tumors treated with saline alone. CTNNB1, $\beta$-catenin; 5 -FU, 5 -fluorouracil.

\section{Results}

Establishment of colon cancer PDX models. Between February 2015 and June 2015, 10 colon cancer specimens were obtained from the Department of General Surgery, Beijing Shijitan Hospital (Beijing, China) and were grafted into nude mice (5 mice per patient). Tumors from 3 patients (30\%) grew in nude mice after 2 months. The success rate of second generation (F2) xenografts was $66.7 \%$ (2/3 patients), and these tumors were passaged through $\geq 5$ generations. The time required for tumor growth was relatively consistent at 3-4 weeks after F2.

Histological and genetic features were maintained following xenograft generation. The histological features of all 10 primary tumors and 2 PDCCX tumors were assessed by pathologists.
Compared with the parental human tumors, PDCCX tumors maintained the original histological characteristics (Fig. 1).

The expression levels of proteins important for colon cancer pathogenesis (SMAD3, ERBB1, c-MET, CDX2, E-cadherin and $\beta$-catenin) were consistent in primary tumors (F0), and first generation (F1) and fifth generation (F5) xenografted tumors (Fig. 2).

Identification of a colon cancer carrying a rare BRAF V600E and CTNNB1 T41A double mutation. Through sequencing analysis of the frequently mutated regions of the most important colon cancer-promoting genes, a patient with colon cancer exhibiting BRAF V600E (Fig. 3A) and CTNNB1 T41A (Fig. 3B) single-nucleotide mutations was identified. This cancer also carried synonymous mutations in the APC, CDH1 and KRAS 
genes (Fig. 3C). The same mutations were detected in the primary cancer tissue and xenografted tumors (F1 and F5).

Drug resistance of colon cancer carrying BRAF V600E and CTNNB1 T41A double mutation. Three classes of frequently used colon cancer therapeutics, 5-FU, oxaliplatin and cetuximab, did not significantly inhibit tumor growth in BRAF V600E/CTNNB1 T41A PDCCX mice (Fig. 4A). The inhibition rate of the three drugs never reached $10 \%$ (Fig. 4B).

\section{Discussion}

In the present study, a PDCCX mouse model was established and a rare colon cancer carrying BRAF V600E/CTNNB1 T41A double mutation was identified. The same mutations were identified in the human primary cancer tissue as well as in the xenografted tumors ( $\leq 5$ generations) in the mice. The histology and colon cancer biomarkers identified in the primary tumors were maintained in F5 engrafted tumors. The BRAF V600E/CTNNB1 T41A PDCCX mouse model was resistant to inhibition induced by 5-FU, oxaliplatin and cetuximab.

PDX have emerged as a reliable tool for the preclinical development of cancer therapeutics, and have been demonstrated to overcome the limitations of cancer cell lines and xenograft animal models $(19,20)$. There have been several publications on the establishment of PDCCX mouse models (21-24). However, these studies did not systemically characterize the genetic alterations of the established PDCCX lines, thereby limiting the applicability of the lines, and also lacked the clarity required for defining the targeted patient population for the therapeutics developed using those lines. The PDCCX line established here had a defined cancer genetic profile and verified oncogenic drivers.

The new PDCCX line reported in the present study carried two well-defined point mutations, BRAF V600E and CTNNB1 T41A, which result in activation of growth factor receptor/Ras/MEK pathways (25) and the Wnt/ $\beta$-catenin pathway (26), respectively, thereby leading to widespread drug resistance. BRAF V600E has been revealed to be associated with resistance to cancer treatments and a poor prognosis (27-29). Wild-type BRAF was shown to be required for response to the EGFR-targeting monoclonal antibodies cetuximab and panitumumab in CRC patients, as BRAF V600E carriers did not respond to these two drugs in a previous study (27). A large-scale phase III trial (PETACC-8 trial) demonstrated that the BRAF V600E mutation led to shorter disease-free survival and overall survival times in patients with microsatellite-stable colon cancers treated with a combination of leucovorin, fluorouracil and oxaliplatin, with or without cetuximab (29). By contrast, activation of the Wnt/ $\beta$-catenin pathway led to increased stemness of cancer cells and drug resistance (30-33). Wnt/ $\beta$-catenin signaling was constitutively activated in breast cancer stem cells, and blockade of the Wnt/ $\beta$-catenin pathway inhibited cancer metastasis (30). Elevated $\beta$-catenin activity promoted carboplatin resistance in the ovarian cancer A2780 cell line (31). Increased nuclear translocation and activation of $\beta$-catenin converted CRC cells into cancer stem cells (32). In a non-small-cell lung cancer cell line carrying the EGFR T790M mutation, inhibition of $\beta$-catenin activity resulted in inhibition of cancer cell stemness and sensitivity to an irreversible tyrosine kinase inhibitor (33). Taken together, these results indicate that activating BRAF and $\beta$-catenin mutations are individual promoters of tumorigenesis and cancer drug resistance, and that the BRAF V600E and CTNNB1 T41A double mutation expands the range of therapeutics that the cancer is resistant to, as well as the complexity and difficulty of developing effective treatments. The PDCCX line established in the present study may provide a reliable tool for developing novel therapeutics that target both the BRAF V600E and CTNNB1 T41A mutations.

Genetic stability of PDX models is critical for their application in the preclinical evaluation of drug efficacy. It was previously reported that the positivity of biomarkers was consistent between parental human gastric cancer tissues and corresponding PDX models (34). Similarly, the biomarker expression was consistent between primary cancer tissues and third-generation PDX of lymphatic and hepatic metastatic colon tumors (35). In the current study, all mutations identified in the primary cancer tissues were present, with similar expression levels, in the F5 PDCCX tumors, indicating that this rare PDCCX model may be beneficial in screening and evaluating therapeutics targeting BRAF and CTNNB1 activating mutations.

In summary, the present study successfully established and characterized a rare PDCCX mouse model carrying BRAF V600E and CTNNB1 T41A activating mutations. Histology, genetic alterations and biomarkers were well-maintained in the PDCCX tumors (F1 and F5). This PDCCX model demonstrated strong resistance to several classes of frequently used colon cancer therapeutics. However, due to the limited number of available cancer samples, we were unable to obtain a sufficient number of PDCCX models carrying either a BRAF V600E or CTNNB1 activating mutation to conclusively determine the interactions between these two colon cancer promoters, which requires consideration in future studies.

\section{Acknowledgements}

The present study was partly supported by the Beijing Municipal Hospital Authority Key Medical Professional Development Program for Minimally Invasive Cancer Therapy (grant no. ZYLX201512).

\section{References}

1. Jemal A, Siegel R, Xu J and Ward E: Cancer statistics, 2010. CA Cancer J Clin 60: 277-300, 2010.

2. Huxley RR, Ansary-Moghaddam A, Clifton P, Czernichow S, Parr CL and Woodward M: The impact of dietary and lifestyle risk factors on risk of colorectal cancer: A quantitative overview of the epidemiological evidence. Int J Cancer 125: 171-180, 2009.

3. Fearon ER: Molecular genetics of colorectal cancer. Annu Rev Pathol 6: 479-507, 2011

4. Cancer Genome Atlas Network: Comprehensive molecular characterization of human colon and rectal cancer. Nature 487: 330-337, 2012.

5. Davies H, Bignell GR, Cox C, Stephens P, Edkins S, Clegg S, Teague J, Woffendin $\mathrm{H}$, Garnett MJ, Bottomley W, et al: Mutations of the BRAF gene in human cancer. Nature 417: 949-954, 2002.

6. Yuan ZX, Wang XY, Qin QY, Chen DF, Zhong QH, Wang L and Wang JP: The prognostic role of BRAF mutation in metastatic colorectal cancer receiving anti-EGFR monoclonal antibodies: A meta-analysis. PLoS One 8: e65995, 2013. 
7. Kolligs FT, Bommer G and Göke B: Wnt/beta-catenin/tcf signaling: A critical pathway in gastrointestinal tumorigenesis. Digestion 66: 131-144, 2002.

8. Lei S, Dubeykovskiy A, Chakladar A, Wojtukiewicz L and Wang TC: The murine gastrin promoter is synergistically activated by transforming growth factor-beta/Smad and Wnt signaling pathways. J Biol Chem 279: 42492-42502, 2004.

9. Guo RJ, Huang E, Ezaki T, Patel N, Sinclair K, Wu J, Klein P, Suh ER and Lynch JP: Cdx1 inhibits human colon cancer cell proliferation by reducing beta-catenin/T-cell factor transcriptional activity. J Biol Chem 279: 36865-36875, 2004.

10. Ripple MJ, Parker Struckhoff A, Trillo-Tinoco J, Li L, Margolin DA, McGoey R and Del Valle L: Activation of c-Myc and Cyclin D1 by JCV T-Antigen and $\beta$-catenin in colon cancer. PLoS One 9: e106257, 2014.

11. Lian J, Tang J, Shi H, Li H, Zhen T, Xie W, Zhang F, Yang Y and Han A: Positive feedback loop of hepatoma-derived growth factor and $\beta$-catenin promotes carcinogenesis of colorectal cancer. Oncotarget 6: 29357-29374, 2015.

12. Wang R, Sun Q, Wang P, Liu M, Xiong S, Luo J, Huang H, Du Q, Geller DA and Cheng B: Notch and Wnt/ $\beta$-catenin signaling pathway play important roles in activating liver cancer stem cells. Oncotarget 7: 5754-5768, 2016.

13. Pandita A,Aldape KD,Zadeh G, Guha A and James CD: Contrasting in vivo and in vitro fates of glioblastoma cell subpopulations with amplified EGFR. Genes Chromosomes Cancer 39: 29-36, 2004.

14. Vescovi AL, Galli R and Reynolds BA: Brain tumour stem cells. Nat Rev Cancer 6: 425-436, 2006.

15. Sasai K, Romer JT, Lee Y, Finkelstein D, Fuller C, McKinnon PJ and Curran T: Shh pathway activity is down-regulated in cultured medulloblastoma cells: Implications for preclinical studies. Cancer Res 66: 4215-4222, 2006.

16. De Witt Hamer PC, VanTilborg AA, Eijk PP, Sminia P, Troost D, Van Noorden CJ, Ylstra B and Leenstra S: The genomic profile of human malignant glioma is altered early in primary cell culture and preserved in spheroids. Oncogene 27: 2091-2096, 2008.

17. Clement V, Sanchez P, de Tribolet N, Radovanovic I and Ruiz Altaba A: HEDGEHOG-GLI1 signaling regulates human glioma growth, cancer stem cell self-renewal, and tumorigenicity. Curr Biol 17: 165-172, 2007.

18. Daniel VC, Marchionni L, Hierman JS, Rhodes JT, Devereux WL, Rudin CM, Yung R, Parmigiani G, Dorsch M, Peacock CD and Watkins DN: A primary xenograft model of small-cell lung cancer reveals irreversible changes in gene expression imposed by culture in vitro. Cancer Res 69: 3364-3373, 2009.

19. Tentler JJ, Tan AC, Weekes CD, Jimeno A, Leong S, Pitts TM, Arcaroli JJ, Messersmith WA and Eckhardt SG: Patient-derived tumour xenografts as models for oncology drug development. Nat Rev Clin Oncol 9: 338-350, 2012.

20. Bousquet $G$ and Janin A: Patient-derived xenograft: An adjuvant technology for the treatment of metastatic disease. Pathobiology 83: 170-176, 2016.

21. Fichtner I, Slisow W, Gill J, Becker M, Elbe B, Hillebrand T and Bibby M: Anticancer drug response and expression of molecular markers in early-passage xenotransplanted colon carcinomas. Eur J Cancer 40: 298-307, 2004.

22. GuenotD, Guérin E, Aguillon-Romain S,Pencreach E, Schneider A, Neuville A, Chenard MP, Duluc I, Du Manoir S, Brigand C, et al: Primary tumour genetic alterations and intra-tumoral heterogeneity are maintained in xenografts of human colon cancers showing chromosome instability. J Pathol 208: 643-652, 2006.
23. Dangles-Marie V, Pocard M, Richon S, Weiswald LB, Assayag F Saulnier P, Judde JG, Janneau JL, Auger N, Validire P, et al: Establishment of human colon cancer cell lines from fresh tumors versus xenografts: Comparison of success rate and cell line features. Cancer Res 67: 398-407, 2007.

24. Linnebacher M, Maletzki C, Ostwald C, Klier U, Krohn M, Klar E and Prall F: Cryopreservation of human colorectal carcinomas prior to xenografting. BMC Cancer 10: 362, 2010.

25. Swaika A, Crozier JA and Joseph RW: Vemurafenib: An evidence-based review of its clinical utility in the treatment of metastatic melanoma. Drug Des Devel Ther 8: 775-787, 2014.

26. Lasota J, Felisiak-Golabek A, Aly FZ, Wang ZF, Thompson LD and Miettinen M: Nuclear expression and gain-of-function $\beta$-catenin mutation in glomangiopericytoma (sinonasal-type hemangiopericytoma): Insight into pathogenesis and a diagnostic marker. Mod Pathol 28: 715-720, 2015.

27. Di Nicolantonio F, Martini M, Molinari F, Sartore-Bianchi A, Arena S, Saletti P, De Dosso S, Mazzucchelli L, Frattini M, Siena S and Bardelli A: Wild-type BRAF is required for response to panitumumab or cetuximab in metastatic colorectal cancer. J Clin Oncol 26: 5705-5712, 2008.

28. Mori Y,Nagasaka T, Mishima H, Umeda Y, Inada R, Kishimoto H, Goel A and Fujiwara T: The rare BRAF VK600-601E mutation as a possible indicator of poor prognosis in rectal carcinoma-a report of a case. BMC Med Genet 16: 1, 2015.

29. Taieb J, Zaanan A, Le Malicot K, Julié C, Blons H, Mineur L, Bennouna J, Tabernero J, Mini E, Folprecht G, et al: Prognostic effect of BRAF and KRAS mutations in patients with stage III colon cancer treated with leucovorin, fluorouracil, and oxaliplatin with or without cetuximab: A post hoc analysis of the PETACC-8 trial. JAMA Oncol 14: 1-11, 2016.

30. Jang GB, Kim JY, Cho SD, Park KS, Jung JY, Lee HY, Hong IS and Nam JS: Blockade of Wnt/ $\beta$-catenin signaling suppresses breast cancer metastasis by inhibiting CSC-like phenotype. Sci Rep 5: 12465, 2015.

31. Barghout SH, Zepeda N, Xu Z, Steed H, Lee CH and Fu Y: Elevated $\beta$-catenin activity contributes to carboplatin resistance in A2780cp ovarian cancer cells. Biochem Biophys Res Commun 468: 173-178, 2015.

32. Wangpu X, Yang X, Zhao J, Lu J, Guan S, Lu J, Kovacevic Z, Liu W, Mi L, Jin R, et al: The metastasis suppressor, NDRG1, inhibits 'stemness' of colorectal cancer via down-regulation of nuclear $\beta$-catenin and CD44. Oncotarget 6: 33893-33911, 2015.

33. Togashi Y, Hayashi H, Terashima M, de Velasco MA, Sakai K, Fujita Y, Tomida S, Nakagawa K and Nishio K: Inhibition of $\beta$-Catenin enhances the anticancer effect of irreversible EGFR-TKI in EGFR-mutated non-small-cell lung cancer with a T790M mutation. J Thorac Oncol 10: 93-101, 2015.

34. Zhang T, Zhang L, Fan S, Zhang M, Fu H, Liu Y, Yin X, Chen H, Xie L, Zhang J, et al: Patient-derived gastric carcinoma xenograft mouse models faithfully represent human tumor molecular diversity. PLoS One 10: e0134493, 2015.

35. Jin K, Li G, Cui B, Zhang J, Lan H, Han N, Xie B, Cao F, He K, Wang $\mathrm{H}$, et al: Assessment of a novel VEGF targeted agent using patient-derived tumor tissue xenograft models of colon carcinoma with lymphatic and hepatic metastases. PLoS One 6: e28384, 2011. 